\title{
Reconstructive Modalities According to Aesthetic Consideration of Subunits of the Cheek after Wide Excision of Skin Cancer
}

Joo Hyun Kim, Hii Sun Jeong, Byeong Ho Lee, Min Su Jung, Seong Hoon Park, Kyoung Seok Tak, In Suck Suh

Department of Plastic and Reconstructive Surgery, Kangnam Sacred Heart Hospital, Hallym University College of Medicine, Seoul, Korea
This article was presented as a poster at the 70th Congress of the Korean Society of Plastic and Reconstructive Surgeons on Nov 9, 2012.

No potential conflict of interest relevant to this article was reported.
Background Defects of the cheek present a reconstructive challenge because of the visibility of the site, as well as the limited local tissue supply. In addition, the cheek abuts several structures with expressive function, such as the eye, mouth, and surrounding facial musculature. This report describes a system of classifying cheek defects according to tumor size and location and present corresponding reconstruction techniques used to accomplish three-dimensional restoration of all missing components and adequate texture matching.

Methods From 2008 to 2013, 27 patients with cheek defects resulting from skin cancer excision were treated. According to the size of the defect, location on the cheek, and relationships to adjacent structures and existing skin tension lines, different surgical methods were applied.

Results The procedures used for cheek reconstruction included direct closure, Limberg flap, $\mathrm{V}-\mathrm{Y}$ advancement flap, slide-swing flap, cheek rotation flap, and full-thickness skin graft. All flaps healed well without major complications, and no cancer recurrence was detected during follow-up.

Conclusions The anatomic classification of skin cancers and surgical protocols described in this report is simple and appropriate for reconstruction involving the cheek. Wide excision of skin cancer and appropriate, relatively easy-to-perform flaps based on this classification system can successfully produce safe and aesthetically-pleasing surgical outcomes.

Keywords Cheek, Esthetics, Reconstructive surgical procedures, Skin neoplasms, Surgical flaps

\section{INTRODUCTION}

The incidence of skin cancer is increasing worldwide, especially in elderly populations. This may be attributed to increased exposure

Received: Jan 29, 2016 Revised: Feb 17, 2016 Accepted: Feb 18, 2016 Correspondence: In Suck Suh Department of Plastic and Reconstructive Surgery, KangNam Sacred Heart Hospital, Hallym University College of Medicine, 1 Singil-ro, Yeongdeungpo-gu, Seoul 07441, Korea.

E-mail: sismdps@hallym.or.kr

Copyright @ 2016 The Korean Society for Aesthetic Plastic Surgery.

This is an Open Access article distributed under the terms of the Creative Commons Attribution Non-Commercial License (http://creativecommons.org/licenses/by-nc/3.0/) which permits unrestricted non-commercial use, distribution, and reproduction in any medium, provided the original work is properly cited. $\quad w w w . e-a a p s . o r g$ to sunlight and outdoor activities, changes in fashion, and/or the rising age of the population. Strong ultraviolet (UV) ray exposure in childhood and adolescence increases the risk of basal cell carcinoma (BCC), whereas chronic UV exposure under the age of 10 can lead to squamous cell carcinoma (SCC). Malignant melanoma is the fastest increasing skin cancer. Its incidence is closely related to skin color and geographical factors [1-3].

The cheek is a highly visible and important part of a person's countenance [4]. Anatomical subunits of the cheek are generally the main factor to consider when choosing the coverage strategy for cheek reconstruction, including the selection and design of local flaps. When reconstructing skin and soft tissue defects caused by skin cancer, direct closure is ideal, as it can create harmony in color and texture with the surrounding tissue. However, in the case 
of skin cancer, which requires excision with a wide safety margin that includes normal tissue, direct closure is often not appropriate. In these cases, a local flap that allows color and texture matching with the surrounding tissue is preferred. In cheek reconstruction, a primary goal is to avoid changes in the adjacent structures, and the cheek has the unique feature that most subunits are not supported by underlying bone $[5,6]$. Among the various ways of subdividing nasal subunits, previous authors have subdivided the cheek into several zones to conceal scar tissue [7].

In this study, we evaluated 27 patients treated for primary facial skin cancer over a 5-year period. We classified the cheek defects into four zones and treated the patients on a case-by-case base according to size of defect, location on the cheek, and relationship to adjacent structures and existing skin tension lines.

\section{METHODS}

We conducted a retrospective study of 27 patients with cheek skin cancer who visited our hospital from 2008 to 2013. All authors followed the principles outlined in the Declaration of Helsinki, and ethics approval was obtained from our Institutional Review Board/ Ethics Committee (\#2014-12-180). Written consent for identifiable photos was obtained from each patient prior to publication. Patients who were injured or had considerable traumatic injuries to the ipsilateral face and neck were excluded. The chief complaints of included patients were protruded, prolonged, and growing pigmented or erythematous masses.

We classified the location of the defects into four zones: lower

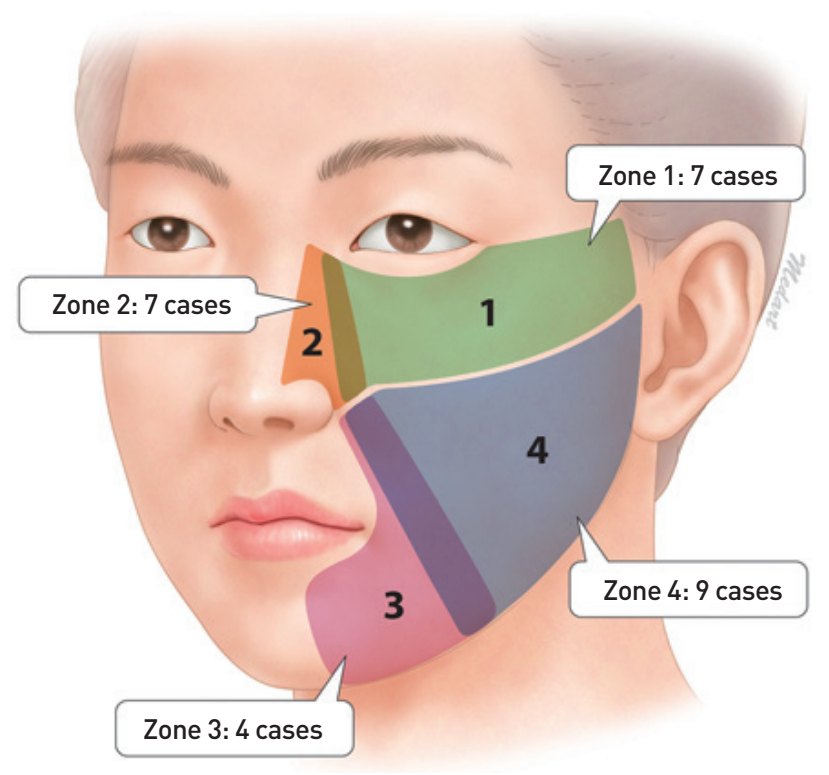

Fig. 1. Schematic diagram. Four subdivisions of the cheek. The surgical defect was located in zone 1 in 7 cases, zone 2 in 7 cases, zone 3 in 4 cases, and zone 4 in 9 cases. eyelid and temple (zone 1), lateral nose (zone 2), nasolabial fold or oral commissure (zone 3), and central cheek (zone 4) (Fig. 1). Zone 1 encompasses the area from the medial canthus to the lateral canthus, including the region extending $1.5 \mathrm{~cm}$ inferior to this, as well as the temple area. Zone 2 begins at the lateral nose and extends 0.5 $\mathrm{cm}$ laterally. Zone 3 begins at the nasolabial fold and corner of the mouth and extends $1 \mathrm{~cm}$ laterally. Otherwise unclassified areas, including the central cheek, comprise zone 4 . The size of the defect, relationship to adjacent structures, and presence of existing skin tension lines were also considered in choosing the most appropriate flap. Some areas could not be covered by a flap because of high tension, so a full thickness skin graft (FTSG) was used to achieve aesthetic reconstruction.

We performed wide excision and set the resection margin at $0.5-1$ $\mathrm{cm}$ for BCC, $1-3 \mathrm{~cm}$ for SCC, and $5 \mathrm{~cm}$ for malignant melanoma. To decrease the recurrence risk, the tumors were removed down to the fascia (which is an anatomical barrier) when the tumor had a deep margin. Appropriate flaps were selected according to skin laxity at the donor site after the wide excision and according to the tension of the flap after covering the defect.

The occurrence of acute and chronic postoperative complications, such as hematoma, inflammation, infection, skin necrosis, or hypertrophic scars, was evaluated. Tumor surveillance was conducted every 3 months after surgery with a physical examination. As well, ultrasonography was performed every 3 months for the first year after surgery, and every 6 months thereafter. The ultrasound evaluations for cancer recurrence were performed by a skilled radiologist. To reduce scar visibility, the scars were managed with tape, silicone gel, and silicone sheets postoperatively.

At 3,6, and 12 months postoperatively, the patients' aesthetic condition and function were examined. Aesthetic assessment was performed by two physicians using the Vancouver Scar Scale (VSS). The VSS consists of four parameters: pigmentation, vascularity, pliability, and height. The highest possible score is 13 , indicating the worst scar condition, whereas a lower score indicates a more desirable scar. Other assessments were functional satisfaction (involving the actions of the facial muscles while closing eyes, smiling, and making other facial expressions), as well as facial asymmetry and difficulties.

\section{RESULTS}

A total of 27 patients were included. Their mean age was 75.6 (5591) years. There were 8 males and 19 females. The cancers were elevated and indurated in 15 patients, and ulcerated and crusting in 12 patients. Pathological examination revealed 13 BCCs, 11 SCCs, and 3 malignant melanomas (Fig. 2). The tumors ranged from 0.5 to $1 \mathrm{~cm}$ in diameter (Table 1).

Seven patients had a defect in zone 1 (lower eyelid and temple), 7 patients in zone 2 (lateral nose), 4 patients in zone 3 (nasolabial 
fold or oral commissure), and 10 patients in zone 4 (central cheek). Five patients had a defect $<2 \mathrm{~cm}^{2}, 10$ patients had a $2-5 \mathrm{~cm}^{2}$ defect, 7 patients had a $5-8 \mathrm{~cm}^{2}$ defect, and 5 patients had a defect $>9 \mathrm{~cm}^{2}$ (Fig. 3). The reconstruction techniques were direct closure (4 cases), FTSG (2 cases), and a local flap. The latter 6 Limberg flaps (Fig. 4), 2 V-Y advancement flaps, 7 slide-swing flaps (Fig. 5), and 6 cheek rotation flaps (Fig. 6, Table 2).

Distal flap necrosis and mild hematomas as acute complications

Table 1. Size of skin cancer

\begin{tabular}{lccc}
\hline & $A^{\text {a) }}$ & $B^{\text {b) }}$ & C $^{\text {c) }}$ \\
\hline Basal cell carcinoma & 3 & 6 & 4 \\
Squamous cell carcinoma & 2 & 7 & 2 \\
Malignant melanoma & - & - & 3 \\
\hline
\end{tabular}

${ }^{\text {a) }}$ size $\leqslant 0.5 \times 0.5 \mathrm{~cm}^{2},{ }^{\text {bl }} 0.5 \times 0.5 \mathrm{~cm}^{2}<$ size $\leqslant 1.0 \times 1.0 \mathrm{~cm}^{2},{ }^{\mathrm{c} 1} 1.0 \times 1.0 \mathrm{~cm}^{2}<$ size.

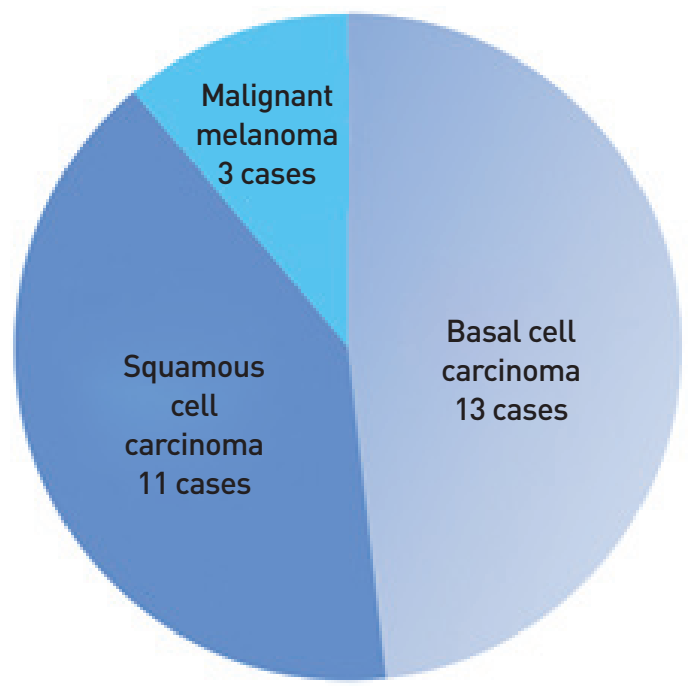

Fig. 2. Pathologic results. Basal cell carcinoma was present in 11 cases, squamous cell carcinoma in 11 cases, and malignant melanoma in 3 cases. occurred in three patients; they healed spontaneously within 1 month. Wound infection, total flap loss, and perioperative mortality did not occur. Hematomas under the flap required surgical evacuation, and patients who were smokers developed minor necrosis at the flap tip. The flaps were all pinkish, similar in color to the surround-

Table 2. Techniques used for cheek reconstruction

\begin{tabular}{lc}
\hline Repair technique & No. of cases (27) \\
\hline Direct closure & 4 \\
Skin graft (full thickness skin graft) & 2 \\
Local flap & \\
Limberg flap & 6 \\
V-Y advancement flap & 2 \\
Slide swing flap & 7 \\
Cheek rotation flap & 6 \\
Total & 27 \\
\hline
\end{tabular}

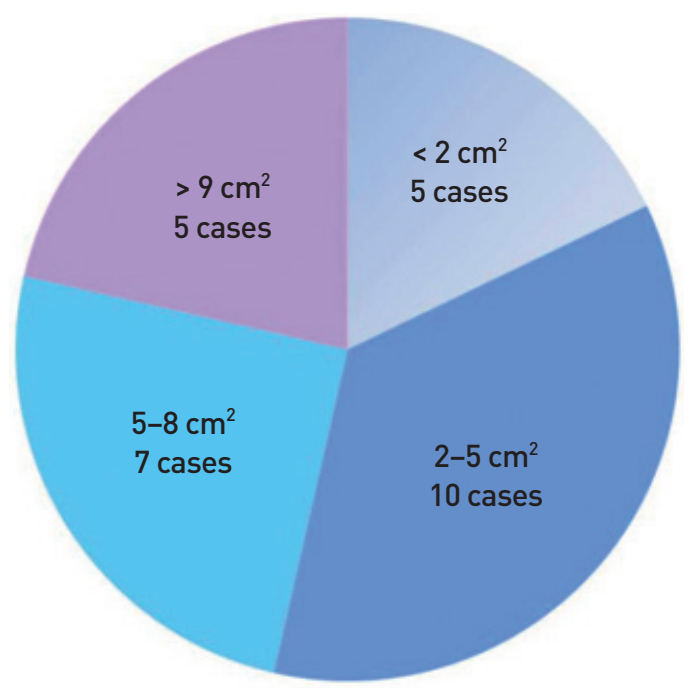

Fig. 3. Defect sizes. Defect size after wide excision of the skin cancer. The defect was $<2 \mathrm{~cm}^{2}$ in 6 cases, $2-5 \mathrm{~cm}^{2}$ in 12 cases, $5-8 \mathrm{~cm}^{2}$ in 8 cases, and $>9 \mathrm{~cm}^{2}$ in 5 cases.
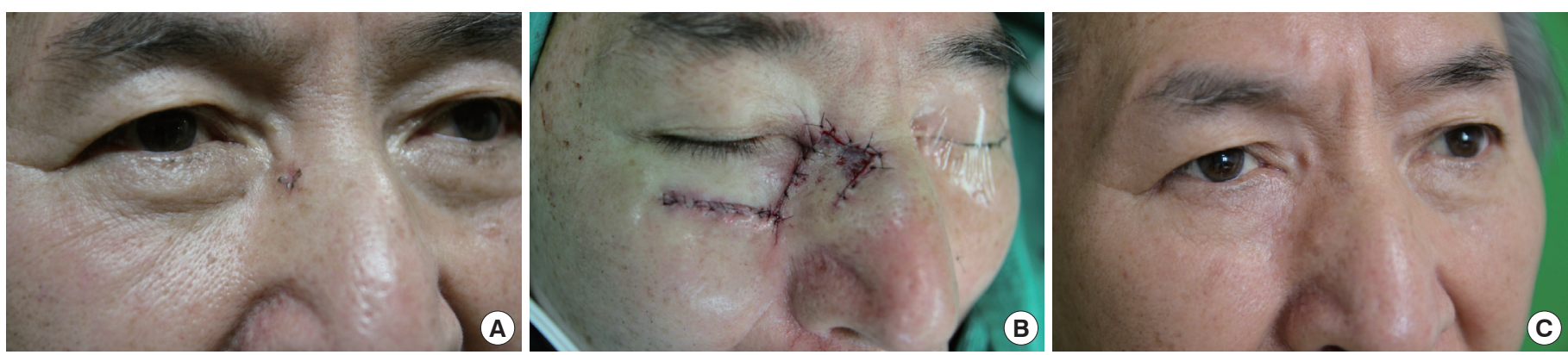

Fig. 4. A case of the Limberg flap. A 69-year-old male with basal cell carcinoma in zone 2. (A) Preoperative view, (B) intraoperative view, and (C) 6 months postoperative view. 
ing tissues, and blanching was easily detected. Almost all patients scored $<3$ on the VSS at 6 months postoperatively; the mean VSS score at this time was $2.56(0-6)$. Functionally, all patients had normal facial expressions and no deformity of key structures. No pa- tient requested a secondary surgery, and most patients were satisfied with their aesthetic results. Furthermore, when monitored closely by ultrasonography for a mean of 76 (24-168) weeks postoperatively, no recurrent cancer was noted in any patient.
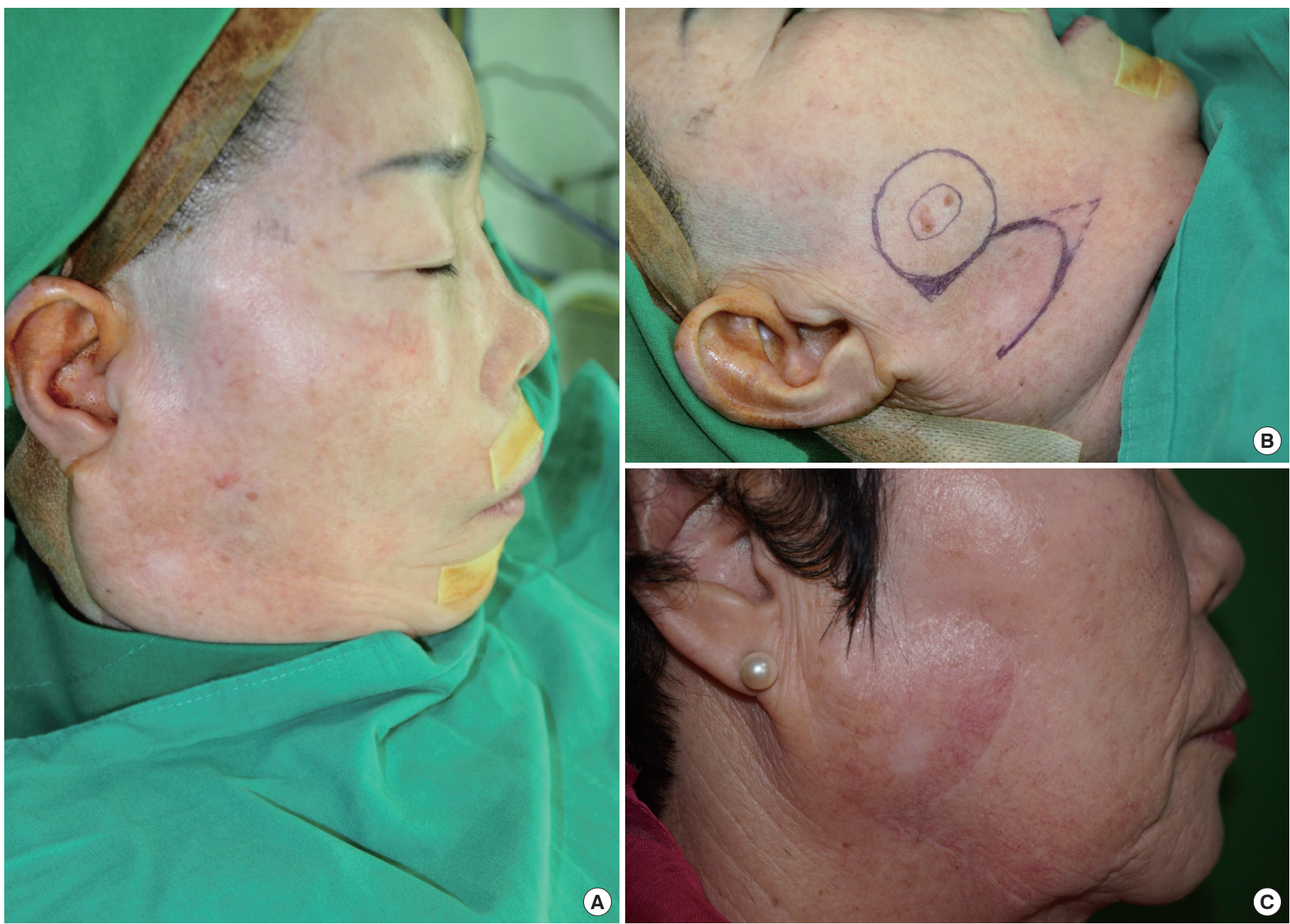

Fig. 5. A case of the slide-swing flap. A 73-year-old female with squamous cell carcinoma in zone 4. (A) Preoperative view, (B) intraoperative view, and (C) 12 months postoperative view.
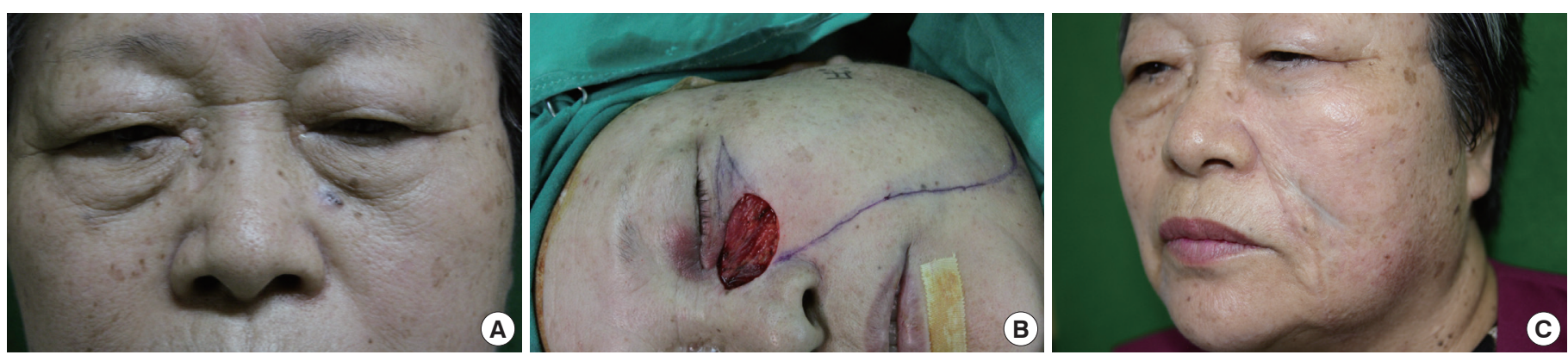

Fig. 6. A case of cheek rotation flap. A 73-year-old female with basal cell carcinoma in zone 1. (A) Preoperative view, (B) intraoperative view, and (C) 6 months postoperative view. 


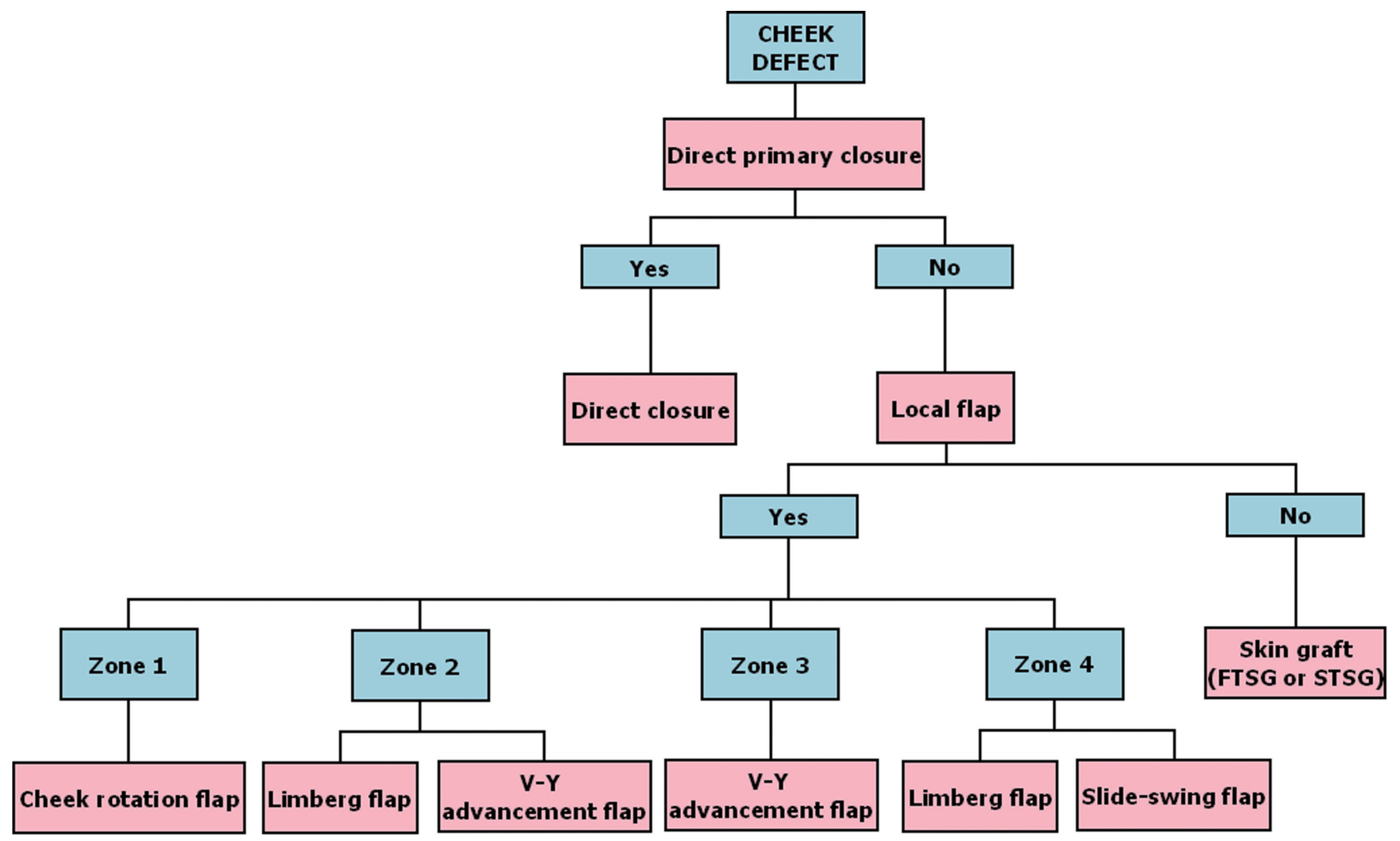

Fig. 7. Treatment algorithm. Most frequent reconstructive modality after skin cancer excision according to a zone classification system based on the cheek defect location. FTSG, full thickness skin graft; STSG, split thickness skin graft.

\section{DISCUSSION}

As the cheek covers a large area of the face and is located near the eyes, mouth, and adjacent facial muscles with expressive functions, a surgeon must be especially careful when performing reconstruction in this area. However, because the cheek has a limited supply of the tissue, a large defect of the cheek is difficult to reconstruct. Moreover, the texture of the cheek varies depending on age and gender. The skin texture of a females cheek is smooth and soft, whereas a male's cheek contains a prominent hair-bearing portion.

As a means of reconstructing skin and soft tissue defects caused by skin cancer excision, direct closure is ideal to preserve harmony in color and texture with the surrounding tissue. However, a wide safety margin is required when excising skin cancer, so direct closure is often impossible without distorting the surrounding key structures, such as the eyelids, nose, and mouth. Direct closure is difficult to perform for a defect $>2 \mathrm{~cm}^{2}$. For larger defects, a local flap is preferred to optimize matching of color and texture with the surrounding tissue.

To achieve both functionally- and aesthetically-satisfying results, cheek reconstruction requires not only functional recovery but also careful three-dimensional recovery of all lost components and suf- ficient tissue matching. As there are no published standards for cheek reconstruction according to defect size and area, we have empirically used many options, including FTSG, local rotation or advancement flaps, regional flaps, tissue expansion, and free tissue transfer [8]. Moreover, skin laxity and the degree of scar formation differ according to race, so a universally applicable algorithm for reconstruction of cheek skin cancer is difficult to establish.

The cheek was divided into three overlapping aesthetic units by Rapstine et al. [7] and Gonzalez-Ulloa et al. [9] zone I included the suborbital region, zone II involved the preauricular region, and zone III encompassed the buco-mandibular region. In the current study, we further subdivided the sections, adding one more area to generate four cheek regions based on anatomical landmarks, which facilitated the creation of a surgical plan of the ideal flap in each area. Choi et al. [10] suggested that a lateral cheek rotation flap is preferred for cheek defects, as it has a similar tissue texture to the defect area and requires less time intraoperatively. During suboptimal reconstruction, elevation, medial and upward rotation, and advancement of a posteroinferiorly-based lateral cheek skin flap is used for coverage, being careful to avoid lower lid ectropion, which can result in corneal exposure [11]. Lower lid ectropion not only potentially affects vision, but it also lowers aesthetic satisfaction. 
In this study, we considered the size, location, and relationship to adjacent tissues of the defect caused by cancer excision and applied different surgical methods accordingly (Fig. 7). For all sizes of defects, direct closure was the first-choice procedure when it was possible to perform without altering the adjacent tissue. Direct closure is best when considering scarring and texture. If this was impossible, we chose a type of local flap, based on various standards. The texture of a local flap is much better than that achieved by a skin graft, although a scar can be seen. Limberg flaps and V-Y advancement flaps were considered in relation to relaxed skin tension line (RSTL). Slide-swing flaps and cheek rotation flaps were chosen based on aesthetic subunits. We selected the incision and flap based on the RSTL of the cheek, as well as the corner of the mouth, and we designed the flap opposite to the RSTL to reduce deformity. If flap coverage was impossible, FTSG was performed.

There are also differences in surgical approach based on the characteristics of each zone. In zone 1, reducing tension that pulled the lower eyelid downward was especially important to prevent lower lid ectropion, so we achieved satisfactory results with a cheek rotation flap through minimizing the tension pulling the lid inferiorly and concealing the lower eyelid scar in an RSTL. For malignancies located in the temple area, care should be taken to avoid deformities of the lateral canthus. Our first choice was the Limberg flap when skin laxity was sufficient. When it was insufficient, an FTSG was performed behind the ear. In zone 2 , which includes the lateral nose, minimizing the tension that pulls the nose laterally should prevent a nose deformity. Limberg and bilobed flaps can minimize nose deformities, but in Asians, the scar caused by a bilobed flap is more prominent than in Caucasians, so we used Limberg or V-Y advancement flap coverage in this area. In zone 3 (from the nasolabial fold to the corner of the mouth), the V-Y advancement flap was used in defects $<2 \mathrm{~cm}^{2}$ and better aesthetic results were achieved by concealing the scars under the nasolabial fold. In zone 4 , which has relatively abundant tissue, direct closure was performed when possible in the lateral cheek area. Limberg and slide swing flaps were used in defects $<2 \mathrm{~cm}^{2}$ if permitted by skin laxity [12,13]. FTSG was performed if there was reduced skin laxity at the malar eminence, which was more common in relatively young patients.

In general, when the use of a local flap was not possible because of poor skin laxity or the possibility of deformity of the adjacent anatomy, a skin graft was used. FTSG was preferred because split thickness skin grafts (STSGs) are more likely to produce secondary contractures, hyperpigmentation, and hypertrophic scars, which makes STSG inferior in terms of aesthetic outcomes.

Direct closure for reconstruction of cheek defects is a technique that must be considered before anything else, as the most natural skin texture is achieved by this technique. From the perspective of size, this technique can be considered if the defect is $<2 \mathrm{~cm}^{2}$, and from the perspective of location, it can be easily applied to zone 4 , which has more space. Also, skin laxity increases as patients become older. Thus, direct closure without altering the surrounding structures, such as the eyes, nose, and ears, was possible in the above circumstances. However, aesthetic problems can arise if direct closure is performed in all instances, as direct closure increases the scar length [14]. Furthermore, direct closure is not commonly used in zone 1 or 2 because of the possibility of ectropion due to deformity involving the eye (in zone 1) or asymmetry due to deformity involving the nose (in zone 2). When performing direct closure, it is important to adjust the closure to coincide with an RSTL to reduce the likelihood of a visible scar.

The Limberg flap is a technique of covering a defect with high tension by transposition of the donor site, which is located around the defect and has relatively good skin. The Limberg flap is designed to cover a 60-degree rhombic defect corresponding to an RSTL. This flap can be used for a lesion with a 3 to $4 \mathrm{~cm}^{2}$ defect size and adequate skin laxity, as is typically found in zone 2 or 4 . The disadvantage of this flap is that additional scar revision could be needed if the patient complains of a dog-ear deformity. However, a trapdoor scar can be relieved by scar management with compression using a silicone sheet. In addition, it may be possible to reduce the size of the defect by undermining and advancing the edges [14] (Fig. 4).

The V-Y advancement flap is a commonly-preferred technique for cheek reconstruction. This flap is superior to rotation flaps because it eliminates the need to revise dog-ears. Also, after closure of a defect, a Y-shaped line can be invisible if it corresponds to an RSTL. Accordingly, it is a particularly ideal flap for zone 3 because the nasolabial fold anatomically hides the scars. This flap is typically used if the defect is $\leq 3 \mathrm{~cm}^{2}$ and close to key structures. However, since it is a type of V-shaped island flap, if insufficient attention is paid to traction, twisting, and similar forces acting on the pedicle, it can easily become devascularized. Therefore, tracing of the pedicle by Doppler ultrasonography is important intraoperatively, and color and blanching of the flap should be closely monitored postoperatively.

A slide-swing flap covers defects by rotating the flap with a combination of transposition and skin sliding and closes the donor site by retracting the surrounding tissue. The slide-swing flap is a way of covering a defect with high tension using tissue with sufficient skin laxity around the defect, as with the Limberg flap. The size of this flap depends on the elasticity of skin, so it is used mainly in zone 4 , which has high elasticity. In addition, it can be useful for relatively large defects. However, tension at the suture site is high, and a trapdoor scar can occur; thus, it is difficult to use in a curved surface $[12,13]$. Nevertheless, a trapdoor scar can be reduced by scar management with compression using a silicone sheet. Accordingly, the slide-swing flap can be useful in zone 4 , which has much excess tissue (Fig. 5).

A cheek rotation flap can cover a wider defect than the other local flaps described in this report because it moves the flap after dis- 
secting a wide area of the cheek. It should be performed by an experienced surgeon, since it requires superficial and delicate dissection in a superficial muscular aponeurotic system to avoid damage to the surrounding structures. It rotates horizontally in the direction from lateral to medial and inferior to superior. The length of cheek incision and range of undermining should be determined by checking whether the flap can cover the defect site at any time during the operation. This flap can be useful, especially in zone 1 and 2 , to avoid ectropion due to vertical tension that draws the lower eyelid downward [10] (Fig. 6).

We evaluated the size and depth of skin cancer with ultrasonography before surgery. Although the cancer did not extend below the subcutaneous fat layer in any of our patients, we chose to reduce the risk of recurrence by sufficient excision involving the fascia, which is considered an anatomical barrier. Furthermore, no recurrence of cancer was detected in any of our patients, despite thorough monitoring by ultrasonography for a mean of 76 weeks postoperatively. Choosing a sufficient surgical resection margin through the use of frozen biopsy examinations intraoperatively and assessing the invasion of tumor toward the surrounding tissue by permanent biopsy postoperatively likely contributed to the lack of cancer recurrence by local invasion, which is a concern when the defect is covered by a local flap.

In conclusion, the anatomic classification of skin cancers and surgical protocols described in this report is simple and appropriate for reconstruction involving the cheek. Although size of the defect, skin laxity, and the relationship to adjacent structures should all be considered when planning reconstructive surgery, as described in the algorithm discussed above, local flaps can be chosen according to the location of the cheek defect to help establish the optimal treatment plan. In situations when skin cancer was completely resected with a wide safety margin, preferential use of the appropriate flap (instead of a skin graft) based on our classification system resulted in satisfactory surgical outcomes, including successful defect closure, aesthetic scar positioning, and minimum postoperative complications.

\section{PATIENT CONSENT}

Patients provided written consent for the use of their images.

\section{REFERENCES}

1. Sng J, Koh D, Siong WC, et al. Skin cancer trends among Asians living in Singapore from 1968 to 2006. J Am Acad Dermatol 2009;61:426-32.

2. Diffey BL, Langtry JA. Skin cancer incidence and the ageing population. Br J Dermatol 2005;153:679-80.

3. Geller AC, Annas GD. Epidemiology of melanoma and nonmelanoma skin cancer. Semin Oncol Nurs 2003;19:2-11.

4. Rashid M, Sarwar S, Hameed S, et al. Experience with lateral cheek rotation flap for the reconstruction of medial cheek soft tissue defects. J Pak Med Assoc 2006;56:227-30.

5. Chandawarkar RY, Cervino AL. Subunits of the cheek: an algorithm for the reconstruction of partial-thickness defects. Br J Plast Surg 2003; 56:135-9.

6. Furr MC, Wang TD. Complex local flap design in cheek reconstruction. Oper Tech Otolaryngol Head Neck Surg 2011;22:53-8.

7. Rapstine ED, Knaus WJ 2nd, Thornton JF. Simplifying cheek reconstruction: a review of over 400 cases. Plast Reconstr Surg 2012;129: 1291-9.

8. Kawashima T, Yamada A, Ueda K, et al. Tissue expansion in facial reconstruction. Plast Reconstr Surg 1994;94:944-50.

9. Gonzalez-Ulloa M, Castillo A, Stevens E, et al. Preliminary study of the total restoration of the facial skin. Plast Reconstr Surg (1946) 1954; 13:151-61.

10. Hashmi ZA, Bashir G, Alamsher, et al. Reconstruction of postleishmaniasis scar with lateral cheek rotation flap. J Coll Physicians Surg Pak 2003;13:405-7.

11. Mustardé JC. Repair and reconstruction in the orbital region. Edinburgh, UK: Churchill Livingstone; 1966.

12. Choi SM, Suh IS, Ha JW. The comparison of the clinical applicability of double Z-rhomboid plasty and slide swing plasty. Arch Aesthetic Plast Surg 1966;2:24-31.

13. Borges AF. The rhombic flap. Plast Reconstr Surg 1981;67:458-66.

14. Strauch B, Vasconez LO, Hall-Findlay EJ, et al. Grabb's encyclopedia of flaps: Head and neck. 3rd ed. Philadelphia, PA: Lippincott Williams and Wilkins; 2008. 